\title{
Neoadjuvant chemotherapy in advanced ovarian cancer: optimal patient selection and response evaluation
}

\author{
Jae Hyun Cho, Seik Kim, Yong Sang Song \\ Department of Obstetrics and Gynecology, Seoul National University College of Medicine, Seoul, Republic of Korea \\ Contributions: (I) Conception and design: YS Song; (II) Administrative support: YS Song; (III) Provision of study materials or patients: S Kim; (IV) \\ Collection and assembly of data: JH Cho; (V) Data analysis and interpretation: JH Cho; (VI) Manuscript writing: All authors; (VII) Final approval of \\ manuscript: All authors. \\ Correspondence to: Yong Sang Song, MD, PhD. Department of Obstetrics and Gynecology, Seoul National University College of Medicine, 101 \\ Daehak-Ro, Jongno-Gu, Seoul 03080, Republic of Korea. Email: yssong@snu.ac.kr.
}

\begin{abstract}
Epithelial ovarian cancer (EOC) is the leading cause of death from gynecologic malignancy and is the seventh most common cancer among women worldwide. The primary debulking surgery (PDS) followed by adjuvant chemotherapy (ACT) is a standard treatment of advanced EOC. However, there are still several limitations, such as high recurrence rate and subsequent chemoresistance. To date, many studies have reported that neoadjuvant chemotherapy (NACT) followed by interval debulking surgery (IDS) shows non-inferior survival outcome and is associated with less postoperative morbidity compared with PDS. Therefore, NACT-IDS might be an alternative treatment strategy in advanced EOC. Nevertheless, the efficacy of NACT-IDS and selection of patients who would gain benefit from NACT-IDS are still on debate. To date, several studies have been conducted to predict the response of NACT using various methods, such as imaging studies and molecular biology techniques. In this article, the optimal indications for NACT using various methods will be discussed.
\end{abstract}

Keywords: Neoadjuvant therapies; interval debulking surgery (IDS); ovarian epithelial cancer; prognostic biomarker; cytoreductive surgical procedure

Submitted Oct 19, 2018. Accepted for publication Oct 22, 2018.

doi: $10.21037 /$ cco.2018.10.11

View this article at: http://dx.doi.org/10.21037/cco.2018.10.11

\section{Introduction}

Epithelial malignancies of ovarian, fallopian tube and peritoneal origin demonstrate similar clinical characteristics and behavior. So these malignancies have been categorized together as epithelial ovarian cancer (EOC) in clinical trials or clinical practice. According to the World Health Organization report, the annual incidence of EOC is estimated as 225,500 with 140,200 deaths worldwide, affecting $3.7 \%$ of all female cancers and $4.2 \%$ of cancer deaths (1). Approximately $80 \%$ of EOC cases are diagnosed at advanced stage, the International Federation of Gynaecology and Obstetrics (FIGO) stage III and IV, resulting in poor survival outcome (2). Most patients experience disease relapse within the first 5 years despite primary aggressive treatment, whereas only $20-25 \%$ of cases are cured. Furthermore, the 5-year survival rate of patients with advanced EOC has not been improved in the last decade.

Current standard therapy for patients with advanced EOC is primary debulking surgery (PDS), followed by adjuvant chemotherapy (ACT) with a combination of paclitaxel and carboplatin (3). Complete or optimal cytoreductive surgery, defined as grossly no residual cancer or $<10 \mathrm{~mm}$ of residual disease at the end of surgery, respectively, is known as the most important prognostic factor. Recently, interval debulking surgery (IDS) after a short course of neoadjuvant chemotherapy (NACT) has become an alternative treatment strategy for EOC patients expecting non-optimal cytoreduction during PDS. Several 
randomized controlled trials (RCTs) have reported that patients who underwent NACT-IDS had significantly lower adverse effect and surgical mortality rate after IDS than those who underwent PDS (4). Survival outcomes, such as progression-free survival (PFS) and overall survival (OS), were similar between NACT-IDS and PDS (5-7). The use of NACT gradually increased from 16\% during 2003 to 2010 to $34 \%$ during 2011 to 2012 in stage IIIC disease, and from $41 \%$ to $62 \%$ in stage IV disease (8).

In accordance with the increased use of NACT, the efficacy of NACT-IDS and selection of patients who would gain benefit from NACT-IDS are still on debate. In this review, we will describe the up to date evidence and indications of NACT, using various diagnostic attempts to improve treatment outcomes of NACT.

\section{Clinical trials comparing NACT-IDS with PDS}

\section{EORTC 55971 trial}

In 1989, the European Organization for Research and Treatment of Cancer (EORTC) and a group of the Netherlands initiated RCTs comparing PDS with NACTIDS, separately. However, due to biases in patient selection and definition of PDS between groups, the studies did not accrue and were early closed (9). Ten years later, the EORTC and National Cancer Institute of Canada (NCIC) initiated a similar trial which completed accrual in 2006. EORTC 55971 trial was a multicenter, non-inferiority RCT including 668 patients with stage IIIC-IV EOC, randomly assigned to either PDS followed by six cycles of carboplatin and paclitaxel or to NACT with carboplatin and paclitaxel for three cycles followed by IDS and another adjuvant three cycles of carboplatin and paclitaxel (5). Compared with PDS, NACT-IDS had a lower rate of complications after initial surgery, including fewer postoperative deaths $(0.7 \%$ vs. $2.5 \%)$, infections ( $2 \%$ vs. $7 \%$ ), grade $3 / 4$ hemorrhage ( $4 \%$ vs. $7 \%)$ and thrombotic events ( $0 \%$ vs. $2.6 \%)$, and a higher rate of optimal cytoreduction ( $80.6 \%$ vs. $41.6 \%)$. On the other hand, PFS [median, 12 months in both arm; hazard ratio (HR), 1.01; 95\% confidence interval (CI), 0.86-1.17] and OS (median, 29 vs. 30 months; HR, 0.98; $95 \%$ CI, 0.82-1.18) were not significantly different. Interestingly, patients treated with PDS showed a trend toward better OS, compared with those who underwent NACT, if there was no residual disease at the time of surgery (median, 45 vs. 38 months; $\mathrm{P}=0.05$ ) or if there was microscopic residual disease only (median, $32 \mathrm{vs}$.
27 months; $\mathrm{P}=0.05$ ). This trial emphasized potential risks of disease progression and diagnostic imprecision associated with NACT. Approximately, a tenth of NACT-IDS patients was unable to undergo IDS, due to disease progression. Accurate comparison is almost impossible between PDS and NACT-IDS group, because of the incomplete surgical staging of NACT. Therefore, some patients wrongly diagnosed or staged and subsequently subjected to incorrect chemotherapy and unnecessary surgery after NACT. In this study, about $3 \%$ of patients $(n=18)$ experienced changes in their diagnosis after surgery.

This study has a novelty for the large cohort research on NACT but has been criticized for a wide variety of chemotherapy regimens. Only $78 \%$ of patients in PDS cohort and $88 \%$ of patients in NACT-IDS cohort received platinum and taxane-based chemotherapy. Another limitation of this trial is that the selection of patient and the surgical technique are not completely uniformed between research institutes. Above all, the rate of optimal cytoreduction in the PDS was only $42 \%$, suggesting that maximal surgical effort was not uniformly attempted $(7,10)$.

\section{The CHemotherapy OR Upfront Surgery (CHORUS) trial}

This large cohort study was a non-inferiority trial that included 552 patient with advanced EOC [16\% (n=72) with stage IV and $80 \%(\mathrm{n}=355)$ with stage III, respectively] recruited from 87 hospitals in the UK and New Zealand, then randomly assigned to PDS or NACT-IDS $(6,7)$. To reduce biases, all women had a clinical assessment with image studies for advanced EOC then underwent randomization followed by a biopsy. Among patients randomly assigned to PDS, only $24 \%$ of patients achieved optimal cytoreduction surgery. In this study, NACT-IDS shows similar OS (median, 24.5 vs. 22.8 months) and PFS (median, 11.7 vs. 10.3 months), compared to PDS. The HR for death was 0.87 in the NACT-IDS arm (95\% CI, 0.72-1.05), which was not inferior to PDS arm. The low rate of optimal cytoreduction in the PDS arm could reduce the potential survival benefit (11).

\section{fCOG 0602 trial}

Two earlier studies, EORTC55971 and CHORUS, demonstrated non-inferior survival rate of patients treated with NACT, however, they could not evaluate treatment invasiveness of NACT (4). The Japanese Clinical Oncology Group (JCOG) 0602 trial compared PDS followed by eight 
Table 1 Comparison of outcomes of four randomized controlled trials for NACT-IDS vs. PDS

\begin{tabular}{|c|c|c|c|c|c|c|c|}
\hline Clinical trial & Author, year & Enrollment criteria & Study arm & $\begin{array}{l}\text { Patient } \\
\text { number } \\
\text { (eligible) }\end{array}$ & $\begin{array}{l}\text { Median } \\
\text { PFS, } \\
\text { months }\end{array}$ & $\begin{array}{l}\text { Median } \\
\text { OS, } \\
\text { months }\end{array}$ & $\begin{array}{l}\text { Operation } \\
\text { time, } \min \end{array}$ \\
\hline $\begin{array}{l}\text { EORTC } \\
55971\end{array}$ & $\begin{array}{l}\text { Vergote et al., } \\
2010\end{array}$ & $\begin{array}{l}\text { Biopsy-proven stage IIIC or IV } \\
\text { EOC or fine-needle aspirate } \\
\text { proven adenocarcinoma in } \\
\text { patient with advanced EOC } \\
\text { on image; ratio of CA-125/ } \\
\text { CEA > } 25\end{array}$ & $\begin{array}{l}\text { Arm 1: PDS followed by at least } \\
\text { six cycles of platinum-based } \\
\text { chemotherapy }\end{array}$ & 310 & 12 & 29 & 165 \\
\hline \multirow[t]{2}{*}{ CHORUS } & \multirow[t]{2}{*}{$\begin{array}{c}\text { Kehoe et al. } \\
2015\end{array}$} & \multirow[t]{2}{*}{$\begin{array}{l}\text { Stage III or IV EOC based on } \\
\text { image studies and ratio of } \\
\text { CA-125/CEA > } 25 \text { then biopsy } \\
\text { after randomization }\end{array}$} & $\begin{array}{c}\text { Arm 1: PDS followed by six } \\
\text { cycles ACT with triweekly } \\
\text { paclitaxel/cisplatin or carboplatin } \\
\text { alone }\end{array}$ & 276 & 10.7 & 22.6 & 120 \\
\hline & & & $\begin{array}{c}\text { Arm 2: three cycles of NACT } \\
\text { followed by IDS }\end{array}$ & 274 & 12 & 24.1 & 120 \\
\hline JCOG 0602 & $\begin{array}{l}\text { Onda et al., } \\
2016\end{array}$ & $\begin{array}{l}\text { Stage III or IV EOC based on } \\
\text { image studies and cytology. } \\
\begin{array}{c}\text { CA-125 }>200 \mathrm{U} / \mathrm{mL} \text { and CEA } \\
<20 \mathrm{ng} / \mathrm{mL}\end{array}\end{array}$ & $\begin{array}{c}\text { Arm 1: PDS followed by eight } \\
\text { cycles of triweekly paclitaxel/ } \\
\text { carboplatin }\end{array}$ & 130 & NA & NA & 341 \\
\hline
\end{tabular}

ACT, adjuvant chemotherapy; CA-125, cancer antigen 125; CEA, carcinoembryonic antigen; CHORUS, CHemotherapy OR Upfront Surgery trial; CR, complete response; EOC, epithelial ovarian cancer; EORTC, European Organization for the Research and Treatment of Cancer; IDS, interval debulking surgery; JCOG, Japan Clinical Oncology Group; NACT, neoadjuvant chemotherapy; NA, not available; OS, overall survival; PDS, primary debulking surgery; PFS, progression-free survival.

cycles of chemotherapy with four cycles of NACT followed by IDS plus four cycles of chemotherapy (12). In this study, the NACT group showed better surgical qualities and less invasiveness than PDS group: fewer total surgery times per patient (mean 0.86 vs. 1.32), shorter operation time (median, 273 vs. 341 minutes), less resection of abdominal organ $(23.7 \%$ vs. $37.6 \%)$ or and distant metastases $(3.9 \%$ vs. $10.7 \%$ ), less blood loss during surgery (median, 787 vs. 3,235 $\mathrm{mL})$, less albumin transfusion $\mathrm{f}(26.2 \%$ vs. $58.5 \%)$, and less adverse events after surgery (4.6\% vs. 15.0\%) (4).

In many studies, NACT-IDS is associated with higher rate of optimal cytoreduction, lower rate of peri-operative morbidity as well as post-surgical mortality, and better quality of life (QOL) than PDS in patients with advanced EOC (13). Table 1 shows a summary of clinical trials comparing NACT-IDS and PDS. Up to now, many studies suggest that there is no difference of OS rate between the two groups. Table 2 summarizes information of ongoing phase III clinical trials.

\section{Patient selection for NACT}

To date, there is no consensus on which patients with advanced EOC should be offered NACT-IDS or PDS. Poor operative conditions, such as medical comorbidities, a poor performance status, or old age, are common indications 
Table 2 Ongoing phase III RCT for NACT

\begin{tabular}{|c|c|c|c|}
\hline $\begin{array}{l}\text { Characteristics of } \\
\text { clinical trials }\end{array}$ & $\begin{array}{l}\text { Study of Upfront Surgery Versus } \\
\text { Neoadjuvant Chemotherapy in } \\
\text { Patients With Advanced Ovarian } \\
\text { Cancer (SUNNY) }\end{array}$ & $\begin{array}{l}\text { Surgical Complications Related } \\
\text { to Primary or Interval Debulking in } \\
\text { Ovarian Neoplasm (SCORPION) }\end{array}$ & $\begin{array}{l}\text { Paclitaxel and Carboplatin With or } \\
\text { Without Bevacizumab in Treating } \\
\text { Patients With Stage II, Stage III, or } \\
\text { Stage IV Ovarian Epithelial Cancer, } \\
\text { Primary Peritoneal Cancer, or Fallopian } \\
\text { Tube Cancer }\end{array}$ \\
\hline Condition of disease & $\begin{array}{l}\text { Stage IIIC or IV epithelial ovarian } \\
\text { cancer, fallopian tube cancer, or } \\
\text { peritoneal carcinoma }\end{array}$ & Stage IIIC epithelial ovarian cancer & $\begin{array}{l}\text { Stage IIA to IV epithelial ovarian cancer, } \\
\text { fallopian tube cancer, or peritoneal } \\
\text { carcinoma }\end{array}$ \\
\hline Intervention & PDS vs. NACT/IDS & PDS vs. NACT/IDS & $\begin{array}{l}\text { PDS/adjuvant chemotherapy } \\
\text { with bevacizumab vs. NACT with } \\
\text { bevacizumab/IDS }\end{array}$ \\
\hline $\begin{array}{l}\text { Number of estimated } \\
\text { enrollments }\end{array}$ & 456 & 171 & 692 \\
\hline Secondary outcome & $\begin{array}{l}\text { PFS; post-operative complication; } \\
\text { quality of life assessments }\end{array}$ & OS; quality of life assessments & OS; quality of life assessments \\
\hline Status & Recruiting & Active, not recruiting & Active, not recruiting \\
\hline $\begin{array}{l}\text { ClinicalTrials.gov } \\
\text { identifier }\end{array}$ & NCT02859038 & NCT01461850 & NCT01167712 \\
\hline
\end{tabular}

ACT, adjuvant chemotherapy; IDS, interval debulking surgery; NACT, neoadjuvant chemotherapy; OS, overall survival; PDS, primary debulking surgery; PFS, progression-free survival.

Table 3 Current indications of NACT

Biopsy proven advanced epithelial ovarian, peritoneal, and
fallopian tubal cancers
Poor performance status and/or medical condition
CT findings with:
(I) diaphragmatic disease $>2 \mathrm{~cm}$;
(II) extra-peritoneal disease such as lung and brain
(III) multiple liver parenchymal metastases requiring total
(IV) pancreatic head and body metastasis;
(V) involvement of the porta hepatis;
(VI) suprarenal para-aortic lymphadenopathy

CT, computerized tomography; LN, Iymph node; NACT, neoadjuvant chemotherapy.

of NACT. Patients with apparently unresectable disease who are impossible to achieve optimal cytoreduction at PDS are also indicated for NACT. According to literature, unresectable diseases are commonly defined as ascites $>1,000 \mathrm{~mL}$, omental extension to the spleen $>1 \mathrm{~cm}$, liver parenchymal disease $>1 \mathrm{~cm}$, porta hepatis involvement $>1 \mathrm{~cm}$, diaphragmatic disease $>1 \mathrm{~cm}$, peritoneal carcinomatosis $>1 \mathrm{~cm}$, and suprarenal para-aortic lymphadenopathy $>1 \mathrm{~cm}$ (14-21). The indications for NACT were summarized in Table 3 (22). However, the unresectable disease can be defined differently depending on the surgical capability of operators. Therefore, further research is needed to apply the criteria of surgically unresectable disease to the clinical management guidelines $(23,24)$.

Attempts to identify the subset of patients most likely to benefit from NACT are under active investigation. An exploratory analysis of patients recruited to the EORTC 55971 trial was done to determine the factors related to the good prognosis with NACT-IDS rather than PDS (25). In this study, age, WHO performance status, tumor grade, tumor histology, serum CA-125, presence of the pelvic mass, or presence of the omental cake were not prognostic factors for 5 -year survival rates by treatment modality. Interestingly, size of the largest metastatic mass was the only prognostic factor for 5 -year OS following primary surgery. Stage IIIC patients with metastatic tumors $\leq 45 \mathrm{~mm}$ 
showed more benefits from PDS, whereas stage IV patients with metastatic tumors $>45 \mathrm{~mm}$ showed more benefits from NACT-IDS. In stage IIIC patients with large metastatic tumors and in stage IV patients with less extensive metastatic tumors, both treatments were equally effective. However, these results still remain to be determined and explained with further studies.

An RCT study by Meyer et al. provided important evidence for appropriate patient selection for NACT (8). In this study, NACT-IDS arm was associated with shorter OS in stage IIIC disease than PDS arm (median, 33 vs. 43 months; HR, 1.40; 95\% CI, 1.11 to 1.77 ), but not in stage IV disease (median, 31 vs. 36 months; HR, 1.16; $95 \%$ CI, 0.89 to 1.52 ). For patients with stage IIIC and IV disease, NACT-IDS arm was less likely to have $\geq 1 \mathrm{~cm}$ of postoperative residual disease, an intensive care unit admission, or a rehospitalization than PDS arm. However, among patients with stage IIIC disease who achieved microscopic or $\leq 1 \mathrm{~cm}$ of residual disease after surgery, NACT was associated with decreased OS (HR, 1.49; 95\% CI, 1.01 to 2.18).

However, the limitation of this study was that all the patients were surgically staged. Therefore, still questions are left; (I) which strategy is better for the patients with stage IIIc-IV disease, PDS or IDS-NACT, (II) which technique is ideal to evaluate the presence of residual tumor after NACT, CT, PET-CT or others, (III) how many cycles are optimal to achieve the best benefit of NACT before planning IDS (26).

\section{Imaging techniques evaluating response to NACT}

Baseline imaging studies, such as computed tomography (CT) have been used as the standard evaluation tool for status of advanced EOC. In NACT-IDS, objective response to chemotherapy is first evaluated after $2-3$ cycles of chemotherapy. However, it is hard to measure the size of gross tumor mass or diffuse small nodules in a reproducible and precise way for the evaluation of chemotherapy response (27). For the present, tumor response is defined as at least a $30 \%$ decrease of the maximum tumor diameter in solid tumors according to the Response Evaluation Criteria in Solid Tumors (RECIST) which is determined after several cycles of chemotherapy (28). So, patients may undergo multiple cycles of ineffective treatment, and delay opportunities to cure with an alternative therapy. Therefore, specific biomarkers and imaging techniques predicting the response to the chemotherapy should be developed to avoid the complications and increase the efficacy of NACT. The imaging techniques for prediction of the response to NACT will be discussed.

\section{Fluorodeoxyglucose-positron emission tomography (18F-FDG-PET)}

18F-FDG-PET has been used in advanced EOC to improve early detection of response and metastatic site (28). Avril et al. suggested that changes in FDG uptake during early treatment course can predict the effectiveness of NACT and patient outcome. They prospectively conducted the sequential FDG-PET imaging at baseline, after the first cycle and third cycle of NACT in 33 patients. The mean and changes of standardized uptake value (SUV) of tumor over the treatment course were then compared with clinical, histopathologic response and OS. They defined the responder as $20 \%$ decrease in FDG uptake after the first cycle of chemotherapy and 55\% decrease in FDG uptake after the first third of chemotherapy. Only 26 out of 33 patients had an FDG-PET scan after the first cycle of chemotherapy and 15 out of 26 patients were responders after the first cycle of NACT (mean decrease in SUV, $59.5 \% \pm 19 \%$ ) and 11 were non-responders (mean decrease in SUV, $4 \% \pm 13.3 \%$ ) according to the definition. After the third cycle of chemotherapy, on the other hand, the most optimal difference in metabolic responders were 18 patients and non-responders were 15 patients. The overall decrease in SUV in metabolic responders was $50.1 \%$ after the first cycle and $76.2 \%$ after the third cycle of chemotherapy in 26 patients. Maximal metabolic changes $(65.7 \%$ of decrease in SUV) were observed within the first 2 weeks $(16 \pm 4.7$ days $)$ after initiation of chemotherapy in metabolic responders. There was a significant correlation between OS rate and the metabolic response after the first $(\mathrm{P}=0.008)$ and the third cycle $(\mathrm{P}=0.005)$ of chemotherapy. The median OS for metabolic responders after the first cycle of chemotherapy was 38.3 months, compared with 23.1 months for metabolic non-responders, and after the third cycle, the median OS was 38.9 months for metabolic responders compared with 19.7 months for metabolic non-responders.

18 FDG-PET was reported to identify histopathological non-responders from the patients with advanced EOC who underwent platinum-based NACT (29). Vallius et al. recruited 26 patients who had primarily inoperable EOC, treated with NACT, and underwent 18F-FDG-PET/CT imaging before diagnostic laparoscopy after three to four 
cycles of NACT. The relationship between the decreasing rates in omental maximum SUV (SUVmax) from pre- to post-NACT, and surgical finding of omental histopathology was evaluated. After NACT, $64 \%$ decrease (range $16 \%$ to $84 \%$ ) of omental SUVmax was associated with histopathological response (OR, 0.9; 95\% CI, 0.84-0.97; $\mathrm{P}=0.004)$. However, SUVmax change was not associated with PFS in this study.

18F-FDG-PET may be helpful for choosing the exact time of cytoreductive surgery. Martoni et al. prospectively measured the changes in metabolic activity of the tumor at baseline, postcycle 3 and postcycle 6 of NACT in 46 patients who were inappropriate for PDS (30). Patients underwent surgery after 6 cycles of NACT and their histopathological response was compared with percentage changes in the SUVmax. They found that in patients with $100 \%$ decrease of SUVmax at 3 cycles, $88 \%$ achieved complete pathological response and no residual disease in debulking surgery, whereas for those patients with a $<100 \%$ decrease in SUVmax at 3 cycles, only $24 \%$ were pathological responders at the end of 6 cycles of NACT. This study suggests that patients who have better metabolic response may get treatment benefit from completing 6 cycles of NACT prior to IDS. The patients with a partial metabolic response lesion could have treatment benefit of IDS after 3 cycles.

\section{Functional magnetic resonance imaging (MRI)}

Functional MRI, such as diffusion-weighted imaging (DWI) and dynamic contrast-enhanced (DCE)-MRI, have been used for the assessment of the response to NACT in advanced EOC. DWI use apparent diffusion coefficient (ADC) of MRI with a specific software that quantifiable the molecular diffusion depending on pulsed field gradients. Molecular diffusion reflects biological gradients of tissue components, such as water, and fibers. The unique diffusion patterns can, therefore, reveal microscopic details about tissue architecture, altering in response to chemotherapy. On the other hand, DCE-MRI allows quantification of changes in tissue vascularity, which may be disrupted in response to chemotherapy.

Kyriazi et al. performed a prospective study for 42 cases of primary or recurrent advanced EOC to identify the role of DWI in chemotherapy response (31). To quantify changes in the tumor before and after treatment, they found that responders showed a significant change in histogram parameters of ADC after the first and third cycle of NACT, whereas there were no significant changes in non-responders. The change in the 25 th percentile of the histogram parameters demonstrated a significant predictability of the treatment response, showing better positive predictive value than CA-125 level or RECIST. However, it did not improve the negative predictive value for the identification of non-responders and percentage change in the 25th percentile did not predict 6-month PFS.

Sala $e t$ al. investigated the association of response to NACT with DWI and DCE-MR in 22 patients with primary advanced EOC (32). Responders showed a significant increase in the ADC and volume of the extravascular extracellular space $[\mathrm{V}(\mathrm{e})]$ of the DCE-MR image due to the cytotoxic effect of chemotherapy. But significant changes were not found in omental or peritoneal deposits but only in the primary ovarian lesion. The authors concluded that ADC and $\mathrm{V}(\mathrm{e})$ parameters can be useful markers reflecting the response to platinum-based NACT in the primary ovarian lesion.

\section{Real-time ultrasound elastography}

Elastography is a modality that maps the elastic properties or stiffness of soft tissue using conventional real - time ultrasound equipment. Generally, the color scale varies within a large band spectrum from red, which may indicate hard and barely elastic tissue, to blue, which may indicate soft and highly elastic tissue on elastogram. Xie et al. investigated the findings of transvaginal and transabdominal sonography, followed by elastography and IDS histopathology (33). Their hypothesis was that the stiffness of tissue in response to NACT would become high due to cellular apoptosis. They scored tissue elasticity from 1 (soft) to 4 (stiff) according to stiffness findings on elastogram. Of 24 patients with advanced EOC, the elasticity score was higher in the post-NACT lesions than in the pre-NACT lesions (mean, $3.13 \pm 0.57$ and median 2 vs. mean, $2.04 \pm 0.51$ and median 4). Post-NACT patients with scores of 3 and 4 had a higher rate of optimal cytoreduction than those with scores of 1 and 2 (93.8\% vs. 25.0\%), with $88.2 \%$ sensitivity, $85.7 \%$ specificity, $93.8 \%$ positive predictive value, $75.0 \%$ negative predictive value and $87.5 \%$ accuracy. The authors suggested that elastography is a sensitive tool for the evaluation of NACT efficacy in patients with advanced EOC. However, due to the limited sensitivity of ultrasound evaluating advanced EOC, it is challenging to apply the elastography to clinical practices. So further studies on appropriate use are needed. 


\section{Biomarkers}

\section{Cancer antigen 125 (CA-125)}

CA-125 is the most widely used serum tumor marker for EOC, and decreasing CA-125 levels are correlated with the response to chemotherapy (34), whereas normalization of CA-125 prior to IDS is associated with improved survival with NACT-IDS. Absolute CA-125 levels and CA-125 regression are known to be predicting outcome, postoperatively (35). Sixty-three patients were evaluated to know whether CA-125 regression is used as a prognostic indicator and predictor of optimal cytoreduction at IDS at NACT setting (36). Authors concluded that CA-125 regression rate predicts the optimal cytoreduction and could be an independent prognostic factor for NACT-IDS.

\section{Immune parameters}

Many clinical and experimental studies have shown that the host immune system plays an important role in the control of cancer growth $(37,38)$. A meta-analysis showed that a high number of tumor-infiltrating lymphocytes (TILs) is significantly associated with an improved survival rate in EOC (39). Mesnage et al. reported that the number of TILs are prognostic indicators not only at diagnosis but also after NACT, retrospectively (40). They compared the TIL number of 83 patients between pre and post-NACT. Following NACT, an overall increase of median TILs from $20 \%$ to $30 \%$ was observed $(\mathrm{P}=0.0005)$. Patients with platinum-free interval $\geq 6$ months had significantly higher post-NACT stromal TILs than those with platinumfree interval $<6$ months ( $28 \%$ vs. $18 \% ; \mathrm{P}=0.026)$. They concluded that high TILs both pre- and post-NACT were independent prognostic factors for PFS (HR 0.49, $\mathrm{P}=0.02$ and HR 0.60, $\mathrm{P}=0.05$, respectively). Research on immune parameters is still on going, possibly promising biomarkers for selecting immunotherapy in patients after NACT.

\section{Tumor cell-free DNA (cfDNA)}

CfDNA is short fragments of nucleic acids containing mutations in the plasma after apoptosis of cancer cells, germline mutations, and somatic mosaic mutations of nonneoplastic DNA. Although less than 1\% of circulating cfDNA comes from cancer cells, the amount increases in patients with advanced disease (41). Many studies have shown that tumor cfDNA can be used for screening, therapeutic decision-making, prognostication, and to predict resistance mechanisms of therapy for cancer patients (42-44). Likewise in patients with EOC, plasma cfDNA can be used as an independent prognostic biomarker and increased tumor cfDNA is associated with poor survival rate which shows better sensitivity and specificity than CA-125 (45).

As a promising novel prognostic biomarker, studies on plasma cfDNA as a response index of NACT have also been conducted. There was a study of genetic variants' analysis for 20 patients with HGSC using pre- and postNACT tumors by next-generation sequencing (NGS) (46). A total of 86 genes had changes in RNA expression after NACT. Including SNPs, 38 genetic variants from 6 genes (TP53, KDR, KIT, PIK3CA, KRAS, and PTEN) were identified in pre-NACT tumors, while 59 variants from 19 genes were detected in the cfDNA. Of the 59 variants in the pre-NACT plasma, only 6 persisted, whereas 33 of 38 specific variants in the pre-NACT tumor persisted. Authors compared 4 genes (PIK3CA I391M, KIT M541L41, $K D R Q 472 H$, and TP53 P72R) which are commonly present at both cfDNA and tumor to evaluate precision and sensitivity of pre- and post-NACT. But all of these 4 genes do not show any significant sensitivity and precision of treatment.

\section{Other biomarkers}

The role of tissue biomarkers in prognostication following NACT is not well established. Recently, the expression of tissue biomarkers such as p53, MIB1, estrogen and progesterone receptors, Her-2/neu, E-cadherin, and Bcl2 was evaluated for NACT biomarkers (47). Following NACT, significant differences in tumor histomorphology were observed in comparison with the chemo-naive neoplasms and low MIB1 was the only significant biomarker representing both treatment outcomes of NACT and survival, and positive ER expression was associated with the poor OS.

\section{Genetic factors affecting NACT}

Homologous recombination (HR) repair pathway is essential to preserve genomic integrity, allowing accurate repair of double-strand DNA breaks, and has been associated with the response to treatment and the overall outcome of patients with HGSC EOC (48). Approximately $15-25 \%$ of EOC patients carry germ-line mutations in $B R C A 1$ or BRCA2 genes (49). BRCA1/2 deficiency is associated with high sensitivity of serous EOC to platinum- 
containing cytotoxic therapy, showing better response and up to $20 \% 5$-year survival rate even for patients with stage IV EOC.

Gorodnova et al. proved that EOC patients with BRCA1/2 germ-line mutation show high sensitivity to platinum-based NACT (50). In their study, of 225 EOC patients, who were treated with platinum-based NACT, 34 BRCA1 and 1 BRCA2 mutation carriers were identified. The complete clinical response was documented in $12 / 35$ (34\%) mutation carriers while in 8/190 (4\%) non-carriers. And the histopathologic response was observed in $16 / 35$ (46\%) women with the germ-line mutation versus $42 / 169$ (24\%) patients with the wild-type genotype. Loss of heterozygosity $(\mathrm{LOH})$ with the residual wild-type BRCA1 allele was observed only in $7 / 24(29 \%)$ post-NACT tumor tissues while in 9/11 (82\%) chemo-naïve BRCA1-tumor tissues. In addition, pre- and post-NACT tumor samples obtained from the same patients showed renovation of BRCA1 heterozygosity in 2 out of 3 sample pairs with $\mathrm{LOH}$ at diagnosis. Through this study, however, the authors did not the association of $\mathrm{LOH}$ of $B R C A 1$ with PFS, OS or the optimal cytoreduction.

Lately, Kessous et al. found that several HR genes demonstrate the association of these genes with prognosis (51). They analyzed the expression pattern of RNA from 96 fresh frozen HGOC tumor samples from PDS $(\mathrm{n}=55)$ and NACT-IDS patients $(\mathrm{n}=41)$. In the PDS group, overexpression of six HR genes (C11orf30, NBN, FANCF, FANCC, FANCB, and RAD50) was associated with better prognosis. In the NACT-IDS group, expression of three HR genes (BRCA2, TP53, and FANCB) was associated with prolonged OS and one gene (RADS1) showed association with better OS and PFS.

\section{Conclusions}

NACT shows better optimal cytoreduction and less perioperative morbidity than standard treatment in patients with advanced EOC. Despite many clinical trials, clinical implication of NACT is still controversial. The lack of consensus on NACT was reflected in the surveys of gynecologic society groups: $70 \%$ of the European Society of Gynecological Oncology (ESGO) members felt the evidence was sufficient to recommend NACT (52). While, in the selected population primarily based on the US, $82 \%$ of Society of Gynecologic Oncologist (SGO) members did not feel there was an evidence to justify the administration of NACT in EOC in the survey of 2010 (53). Even in the follow-up survey conducted 5 years later, although fewer respondents feel evidence is insufficient to support use of NACT/IDS (68\% and $82 \%$ in 2015 and 2010, respectively), most SGO members still do not consider the evidence to be sufficient to support regular use of NACT (79\%) (54). Thus, more studies are warranted to select suitable patients for NACT.

In this review, we discussed the evidence and indications of NACT with various novel diagnostic tools. Previously published studies show that the patients receiving NACT were mainly evaluated by radiologic examination. Because these studies could cause deviated interpretation of clinical outcome, many physicians have questioned the usefulness of NACT. Recently, many groups have tried to overcome this paradigm through a series of advanced imaging and molecular biologic techniques. 18F-FDG-PET and functional MRI, such as DWI and DCE-MRI, have been proven to be a useful tool for screening candidates for NACT and timing of IDS. In addition, the development of molecular biology enabled the discovery of novel biomarkers, such as cfDNA, TILs, and MIB1 showing more precise prediction of response and prognosis than the conventional biomarkers. Over-expression of HR DNA repair pathways, such as C11orf30, NBN, FANCF, FANCC, $F A N C B$, and RAD50, as well as BRCA, were found to be associated with the improved outcome of NACT.

It is the era of personalized medicine. So far, many studies have evaluated the indications and efficacy of NACT based on the large-scale group study. However, the results are still ambiguous. Although many personalized treatments on EOC are underway, unfortunately, well designed prospective study of NACT is still in the early phase. NACT is clearly a helpful treatment option for patients with advanced EOC, and more researches are warranted to find out the appropriate candidates for NACT.

\section{Acknowledgements}

None.

\section{Footnote}

Conflicts of Interest: The authors have no conflicts of interest to declare.

\section{References}

1. Minig L, Zorrero C, Iserte PP, et al. Selecting 
the best strategy of treatment in newly diagnosed advanced-stage ovarian cancer patients. World J Methodol 2015;5:196-202.

2. Seidman JD, Yemelyanova A, Cosin JA, et al. Survival rates for international federation of gynecology and obstetrics stage III ovarian carcinoma by cell type: a study of 262 unselected patients with uniform pathologic review. Int J Gynecol Cancer 2012;22:367-71.

3. Du Bois A, Pfisterer J. Future options for first-line therapy of advanced ovarian cancer. Int J Gynecol Cancer 2005;15 Suppl 1:42-50.

4. Onda T, Satoh T, Saito T, et al. Comparison of treatment invasiveness between upfront debulking surgery versus interval debulking surgery following neoadjuvant chemotherapy for stage III/IV ovarian, tubal, and peritoneal cancers in a phase III randomised trial: Japan Clinical Oncology Group Study JCOG0602. Eur J Cancer 2016;64:22-31.

5. Vergote I, Trope CG, Amant F, et al. Neoadjuvant chemotherapy or primary surgery in stage IIIC or IV ovarian cancer. N Engl J Med 2010;363:943-53.

6. Kehoe S, Hook J, Nankivell M, et al. Primary chemotherapy versus primary surgery for newly diagnosed advanced ovarian cancer (CHORUS): an open-label, randomised, controlled, non-inferiority trial. Lancet 2015;386:249-57.

7. Vergote I, Leunen K, Amant F. Primary surgery or neoadjuvant chemotherapy in ovarian cancer: what is the value of comparing apples with oranges? Gynecol Oncol 2012;124:1-2.

8. Meyer LA, Cronin AM, Sun CC, et al. Use and Effectiveness of Neoadjuvant Chemotherapy for Treatment of Ovarian Cancer. J Clin Oncol 2016;34:3854-63.

9. van der Burg ME, van Lent $M$, Buyse $M$, et al. The effect of debulking surgery after induction chemotherapy on the prognosis in advanced epithelial ovarian cancer. Gynecological Cancer Cooperative Group of the European Organization for Research and Treatment of Cancer. N Engl J Med 1995;332:629-34.

10. Chi DS, Bristow RE, Armstrong DK, et al. Is the easier way ever the better way? J Clin Oncol 2011;29:4073-5.

11. Chi DS, Eisenhauer EL, Zivanovic O, et al. Improved progression-free and overall survival in advanced ovarian cancer as a result of a change in surgical paradigm. Gynecol Oncol 2009;114:26-31.

12. Onda T, Matsumoto K, Shibata T, et al. Phase III trial of upfront debulking surgery versus neoadjuvant chemotherapy for stage III/IV ovarian, tubal and peritoneal cancers: Japan Clinical Oncology Group Study JCOG0602. Jpn J Clin Oncol 2008;38:74-7.

13. Yang L, Zhang B, Xing G, et al. Neoadjuvant chemotherapy versus primary debulking surgery in advanced epithelial ovarian cancer: A meta-analysis of perioperative outcome. PLoS One 2017;12:e0186725.

14. Huober J, Meyer A, Wagner U, et al. The role of neoadjuvant chemotherapy and interval laparotomy in advanced ovarian cancer. J Cancer Res Clin Oncol 2002;128:153-60.

15. Steed H, Oza AM, Murphy J, et al. A retrospective analysis of neoadjuvant platinum-based chemotherapy versus upfront surgery in advanced ovarian cancer. Int J Gynecol Cancer 2006;16 Suppl 1:47-53.

16. Nelson BE, Rosenfield AT, Schwartz PE. Preoperative abdominopelvic computed tomographic prediction of optimal cytoreduction in epithelial ovarian carcinoma. J Clin Oncol 1993;11:166-72.

17. Kuhn W, Rutke S, Spathe K, et al. Neoadjuvant chemotherapy followed by tumor debulking prolongs survival for patients with poor prognosis in International Federation of Gynecology and Obstetrics Stage IIIC ovarian carcinoma. Cancer 2001;92:2585-91.

18. Schwartz PE, Rutherford TJ, Chambers JT, et al. Neoadjuvant chemotherapy for advanced ovarian cancer: long-term survival. Gynecol Oncol 1999;72:93-9.

19. Schwartz PE, Chambers JT, Makuch R. Neoadjuvant chemotherapy for advanced ovarian cancer. Gynecol Oncol 1994;53:33-7.

20. Mazzeo F, Berliere M, Kerger J, et al. Neoadjuvant chemotherapy followed by surgery and adjuvant chemotherapy in patients with primarily unresectable, advanced-stage ovarian cancer. Gynecol Oncol 2003;90:163-9.

21. Vergote IB, De Wever I, Decloedt J, et al. Neoadjuvant chemotherapy versus primary debulking surgery in advanced ovarian cancer. Semin Oncol 2000;27:31-6.

22. Lim MC, Song YJ, Seo S, et al. Residual Cancer Stem Cells after Interval Cytoreductive Surgery following Neoadjuvant Chemotherapy Could Result in Poor Treatment Outcomes for Ovarian Cancer. Onkologie 2010;33:324-30.

23. Salani R, Axtell A, Gerardi M, et al. Limited utility of conventional criteria for predicting unresectable disease in patients with advanced stage epithelial ovarian cancer. Gynecol Oncol 2008;108:271-5.

24. Lim MC, Yoo HJ, Song YJ, et al. Survival outcomes after 


\section{Page 10 of 11}

extensive cytoreductive surgery and selective neoadjuvant chemotherapy according to institutional criteria in bulky stage IIIC and IV epithelial ovarian cancer. J Gynecol Oncol 2017;28:e48.

25. van Meurs HS, Tajik P, Hof MH, et al. Which patients benefit most from primary surgery or neoadjuvant chemotherapy in stage IIIC or IV ovarian cancer? An exploratory analysis of the European Organisation for Research and Treatment of Cancer 55971 randomised trial. Eur J Cancer 2013;49:3191-201.

26. Vergote IB, Van Nieuwenhuysen E, Vanderstichele A. How to Select Neoadjuvant Chemotherapy or Primary Debulking Surgery in Patients With Stage IIIC or IV Ovarian Carcinoma. J Clin Oncol 2016;34:3827-8.

27. Rockall A, Munari A, Avril N. New ways of assessing ovarian cancer response: metabolic imaging and beyond. Cancer Imaging 2012;12:310-4.

28. Avril N, Sassen S, Schmalfeldt B, et al. Prediction of response to neoadjuvant chemotherapy by sequential F-18-fluorodeoxyglucose positron emission tomography in patients with advanced-stage ovarian cancer. J Clin Oncol 2005;23:7445-53.

29. Vallius T, Peter A, Auranen A, et al. 18F-FDG-PET/CT can identify histopathological non-responders to platinumbased neoadjuvant chemotherapy in advanced epithelial ovarian cancer. Gynecol Oncol 2016;140:29-35.

30. Martoni AA, Fanti S, Zamagni C, et al. [18F]FDG$\mathrm{PET} / \mathrm{CT}$ monitoring early identifies advanced ovarian cancer patients who will benefit from prolonged neoadjuvant chemotherapy. Q J Nucl Med Mol Imaging 2011;55:81-90.

31. Kyriazi S, Collins DJ, Messiou C, et al. Metastatic ovarian and primary peritoneal cancer: assessing chemotherapy response with diffusion-weighted MR imaging--value of histogram analysis of apparent diffusion coefficients. Radiology 2011;261:182-92.

32. Sala E, Kataoka MY, Priest AN, et al. Advanced ovarian cancer: multiparametric MR imaging demonstrates response- and metastasis-specific effects. Radiology 2012;263:149-59.

33. Xie $M$, Zhang $X$, Jia Z, et al. Elastography, a sensitive tool for the evaluation of neoadjuvant chemotherapy in patients with high-grade serous ovarian carcinoma. Oncol Lett 2014;8:1652-6.

34. Rustin GJ, Nelstrop AE, McClean P, et al. Defining response of ovarian carcinoma to initial chemotherapy according to serum CA 125 . J Clin Oncol 1996;14:1545-51.

\section{Cho et al. Systemic review on NACT-IDS vS. PDS in advanced EOC}

35. Crawford SM, Peace J. Does the nadir CA125 concentration predict a long-term outcome after chemotherapy for carcinoma of the ovary? Ann Oncol 2005;16:47-50.

36. Vasudev NS, Trigonis I, Cairns DA, et al. The prognostic and predictive value of CA-125 regression during neoadjuvant chemotherapy for advanced ovarian or primary peritoneal carcinoma. Arch Gynecol Obstet 2011;284:221-7.

37. Sato E, Olson SH, Ahn J, et al. Intraepithelial CD8+ tumor-infiltrating lymphocytes and a high CD8+/ regulatory $\mathrm{T}$ cell ratio are associated with favorable prognosis in ovarian cancer. Proc Natl Acad Sci 2005;102:18538-43.

38. Clarke B, Tinker AV, Lee CH, et al. Intraepithelial T cells and prognosis in ovarian carcinoma: novel associations with stage, tumor type, and BRCA1 loss. Mod Pathol 2009;22:393-402.

39. Hwang WT, Adams SF, Tahirovic E, et al. Prognostic significance of tumor-infiltrating $\mathrm{T}$ cells in ovarian cancer: a meta-analysis. Gynecol Oncol 2012;124:192-8.

40. Mesnage SJ, Auguste A, Genestie C, et al. Neoadjuvant chemotherapy (NACT) increases immune infiltration and programmed death-ligand 1 (PD-L1) expression in epithelial ovarian cancer (EOC). Ann Oncol 2017;28:651-7.

41. Leon SA, Shapiro B, Sklaroff DM, et al. Free DNA in the Serum of Cancer Patients and the Effect of Therapy. Cancer Res 1977;37:646-50.

42. Bettegowda C, Sausen M, Leary RJ, et al. Detection of circulating tumor DNA in early-and late-stage human malignancies. Sci Transl Med 2014;6:224ra24.

43. Alix-Panabières C, Pantel K. Clinical applications of circulating tumor cells and circulating tumor DNA as liquid biopsy. Cancer Discov 2016;6:479-91.

44. Canzoniero JV, Park BH. Use of cell free DNA in breast oncology. Biochim Biophys Acta 2016;1865:266-74.

45. Kamat AA, Baldwin M, Urbauer D, et al. Plasma Cellfree DNA in Ovarian Cancer: An Independent Prognostic Biomarker. Cancer 2010;116:1918-25.

46. Arend RC, Londoño AI, Montgomery AM, et al. Molecular Response to Neoadjuvant Chemotherapy in High-Grade Serous Ovarian Carcinoma. Mol Cancer Res 2018;16:813-24.

47. Khandakar B, Mathur SR, Kumar L, et al. Tissue Biomarkers in Prognostication of Serous Ovarian Cancer following Neoadjuvant Chemotherapy. Biomed Res Int 2014;2014:401245. 
48. Katsuki Y, Takata M. Defects in homologous recombination repair behind the human diseases: FA and HBOC. Endocr Relat Cancer 2016;23:T19-37.

49. Alsop K, Fereday S, Meldrum C, et al. BRCA Mutation Frequency and Patterns of Treatment Response in BRCA Mutation-Positive Women With Ovarian Cancer: A Report From the Australian Ovarian Cancer Study Group. J Clin Oncol 2012;30:2654-63.

50. Gorodnova TV, Sokolenko AP, Ivantsov AO, et al. High response rates to neoadjuvant platinum-based therapy in ovarian cancer patients carrying germ-line BRCA mutation. Cancer Lett 2015;369:363-7.

51. Kessous R, Octeau D, Klein K, et al. Distinct homologous recombination gene expression profiles after neoadjuvant chemotherapy associated with clinical outcome in patients with ovarian cancer. Gynecol Oncol 2018;148:553-8.

Cite this article as: Cho JH, Kim S, Song YS. Neoadjuvant chemotherapy in advanced ovarian cancer: optimal patient selection and response evaluation. Chin Clin Oncol 2018;7(6):58. doi: 10.21037/cco.2018.10.11
52. Cornelis S, Van Calster B, Amant F, et al. Role of neoadjuvant chemotherapy in the management of stage IIIC-IV ovarian cancer: survey results from the members of the European Society of Gynecological Oncology. Int J Gynecol Cancer 2012;22:407-16.

53. Dewdney SB, Rimel BJ, Reinhart AJ, et al. The role of neoadjuvant chemotherapy in the management of patients with advanced stage ovarian cancer: survey results from members of the Society of Gynecologic Oncologists. Gynecol Oncol 2010;119:18-21.

54. Huelsmann E, Zighelboim I, Ahmed A, et al. The role of neoadjuvant chemotherapy in the management of patients with advanced stage ovarian cancer: Survey results from members of the society of gynecologic oncologists, a 5-year follow-up. Gynecol Oncol Rep 2017;20:47-50. 